\title{
Adaptive Power Flow Prediction Based on Machine Learning
}

\author{
Jingyeong Park ${ }^{1} \oplus$, Daisuke Kodaira ${ }^{2} \oplus$, Kofi Afrifa Agyeman ${ }^{1}$, Taeyoung Jyung ${ }^{3, *}$ and Sekyung Han ${ }^{1,4, *}$ \\ 1 School of Electronic and Electrical Engineering, Kyungpook National University, Daegu 41566, Korea; \\ jkms3815@gmail.com (J.P.); kofiagyeman@knu.ac.kr (K.A.A.) \\ 2 Department of Electrical Engineering, Tokyo University of Science, Tokyo 162-8601, Japan; \\ daisuke.kodaira03@gmail.com \\ 3 Korea Electric Power Corporation Engineering \& Construction (KEPCO E\&C), Gimcheon 39660, Korea \\ 4 Department of Electrical Engineering, Kyungpook National University, Daegu 41566, Korea \\ * Correspondence: tyjyung@kepco-enc.com (T.J.); skhan@knu.ac.kr (S.H.); Tel.: +81-10-3820-8521 (T.J.); \\ +81-10-8928-3752 (S.H.)
}

Citation: Park, J.; Kodaira, D.; Agyeman, K.A.; Jyung, T.; Han, S. Adaptive Power Flow Prediction Based on Machine Learning. Energies 2021, 14, 3842. https://doi.org/ $10.3390 /$ en14133842

Academic Editor: Nicu Bizon

Received: 27 April 2021

Accepted: 23 June 2021

Published: 25 June 2021

Publisher's Note: MDPI stays neutral with regard to jurisdictional claims in published maps and institutional affiliations.

Copyright: (c) 2021 by the authors. Licensee MDPI, Basel, Switzerland. This article is an open access article distributed under the terms and conditions of the Creative Commons Attribution (CC BY) license (https:// creativecommons.org/licenses/by/ $4.0 /)$.

\begin{abstract}
Power flow analysis is an inevitable methodology in the planning and operation of the power grid. It has been performed for the transmission system, however, along with the penetration of the distributed energy resources, the target has been expanded to the distribution system as well. However, it is not easy to apply the conventional method to the distribution system since the essential information for the power flow analysis, say the impedance and the topology, are not available for the distribution system. To this end, this paper proposes an alternative method based on practically available parameters at the terminal nodes without the precedent information. Since the available information is different between high-voltage and low-voltage systems, we develop two various machine learning schemes. Specifically, the high-voltage model incorporates the slack node voltage, which can be practically obtained at the substation, and yields a time-invariant model. On the other hand, the low voltage model utilizes the deviation of voltages at each node for the power changes, subsequently resulting in a time-varying model. The performance of the suggested models is also verified using numerical simulations. The results are analyzed and compared with another power flow scheme for the distribution system that the authors suggested beforehand.
\end{abstract}

Keywords: power flow; distribution network; machine learning; slack node voltage; impedance estimation

\section{Introduction}

The conventional electric power distribution system has a unidirectional power flow that decreases monotonically from substations to the line terminal, making voltage control relatively easy. However, with the growing penetration of distributed energy resources, the possibility of voltage violation has rapidly increased [1-5]. Near the point where distributed generation is connected, there is a high chance that the voltage violates the upper limit. Reversely, the terminal load of the distribution system may suffer a low voltage issue.

The primary method to interpret this problem is power flow analysis, which performs the numerical analysis. This method investigates if the power flow is within the allowable safe operating range for each power facility and if each bus voltage is within the regulated range by considering available types of power system configuration. If a control scheme is to be designed to mitigate the voltage violation, the power flow analysis is essential to evaluate the stability of the voltage. However, the power flow analysis includes many variables and involves complex nonlinear calculation formulas. As there is no general analytical solution, it is calculated through repeated numerical methods, hence taking a long time to derive a convergent solution. Especially, it is tricky to utilize the power flow analysis in a typical optimal control scheme as it is a time-consuming numerical solution [1,6-8]. Moreover, it requires detailed information on the target system, such as 
topology configuration and line impedances of the network, normally not available for the distribution network. Also, the power transactions at all nodes should be available as well. Voltage estimation with numerical methods is sensitive to system parameter values. Therefore, numerical power flow solvers are subject to the inaccuracy of power flow models [9]. These issues emanate from missing knowledge of system parameters and control rules. Especially on the distribution network, data acquisition may not be readily available due to the network topology's unascertained nature [10]. Moreover, knowledge of their existence on the network may not be readily known since they are usually independently owned by consumers [11]. To tackle the problems, some studies have been performed to estimate the state of the system through repetitive calculation by nonlinear weighted least squares (WLS) in the situation where the power values of all loads are not given [12,13]. Yet, this is possible only when the topology and line parameters (impedances) of the distribution system are secured. In practice, however, the information of the low-voltage distribution lines is not managed or databased in most of the country. Thus, it is practically impossible to apply such a state-based method. For such problems, research on calculating the voltage/phase angle for power flow, including many variables and equations with only known quantities, i.e., active power/reactive power, by introducing the artificial neural networks (ANN) [14-17] and Probabilistic Load Flow based on Deep Learning, have been conducted [18]. Since the artificial neural network-based model can guarantee accurate and fast execution time, it can be applied to real-time control of complex power systems. However, the ANN-based models in the previous research are only available when the slack bus voltage is constant; it is not applicable when the slack bus voltage changes. For this reason, it is necessary to learn the known quantity of a load according to the voltage variation of the slack bus as it is not always constant. Another problem is that it is limited only to a specific topology.

To solve this problem, this paper suggests two alternative machine learning (ML) schemes for high voltage and low voltage distribution networks considering the typical configuration of each network, respectively. We commonly consider only the rms magnitude of the voltage rather than the angle values for both models. The purpose of power flow analysis on the distribution system is to check the voltage violation rather than the angle difference itself. In the high-voltage distribution system, the slack voltage is assumed as given information since the slack bus on the high-voltage distribution system is a substation. In a substation, the bus voltage is constantly managed and can be provided as measured information. Apart from the slack bus information, the rest of the information needed for the power flow analysis is only the rms magnitude of the complex power from each node. As a result, all necessary information can be collected in a synchronized manner, and subsequently, the power flow analysis can be performed as a one-shot steady-state calculation without a time-varying variable. On the other hand, the slack bus in a low-voltage distribution system is usually a pole-transformer. In this case, the voltage information for the slack bus usually is not available. Therefore, it is necessary to design the estimation scheme without the slack bus information. In general, however, the steady-state power flow analysis can be performed only when all bus information is present. Even with the machine learning scheme's general flexibility, missing the slack bus information is critical and can yield a vast error. To overcome this defect, we designed the model to include time-varying information. This idea comes from the fact that the slack bus is the entry point of the currents to all the child nodes. Since the slack bus is on the uppermost of the current flow, the same amount of perturbation on any node will cause different results depending on the voltage state of the slack bus. Therefore, it can be expected that the machine learning scheme recognizes the difference of the slack node voltages if it is trained with the deviation information of the node voltages rather than the absolute values.

For both models, mathematical analyses are also performed. By representing the model output as a function of the input parameters, the effectiveness of the model can be validated. In addition, performance analysis is performed by comparing the prediction accuracy with that of the conventional power flow analysis results. It is also compared with 
another alternative power flow solution that has been suggested by the authors [19]. The previous method identified the line impedances and performed the conventional power flow analysis using the estimated impedance information. This method, however, also assumes the constant slack bus voltage, say 1.0 (p.u), and therefore the usage is limited.

The rest of the paper is organized as follows: Section 2 presents the power flow methodology based on machine learning for high and low-voltage distribution systems in detail. In Section 3, the proposed model is evaluated using a test grid.

\section{Machine-Learning Based Power Flow Analysis}

Solving the power flow (PF) problem with machine learning methods yields solutions by surmising the mapping rules between the observed system input and output parameters based on acquired historical system operation data. To predict nodes' rms magnitude of the voltage, machine learning power flow solvers use algorithms to solve by learning the mapping rules from specified variables to node voltages. This section describes a machine-learning algorithm to solve the PF problem under the power network's generic physical knowledge constraints. We do so by defining a machine learning black-box model to predict the PF parameters under a set of defined conditions.

\subsection{Machine-Learning Based Power Flow Solver Problem}

Conventional numerical computation of PF solution attains feasibility by relying on data-driven approaches to predict unknown system parameters such as node impedances. However, assumptions and errors associated with these parameter estimations affect the accuracy of the numerical solution. Numerical solutions are sensitive to disparities in system parameters. A slight bias in the estimated system parameters can induce colossal variance in the estimated numerical solution. Therefore, we devise a new variant of a datadriven power flow model based on machine learning algorithms to mitigate these problems.

Our proposed model focuses on both the HV and LV sides of the distribution network. Whereas the proposed model predicts the node voltages of the HV side of the distribution network, the change in voltage, $\Delta \mathrm{V}$, is predicted for the network's LV side with unknown slack node voltage. The proposed scheme is classified into two models: High-Voltage Machine Learning (HV-ML) and Low-Voltage Machine Learning (LV-ML) models. The remaining section described these models in detail.

\subsection{High-Voltage Machine-Learning ( $H V-M L)$}

The HV-ML model applies to the HV side of the distribution network. Generally, the slack node voltage of the HV side of the distribution network corresponds to the substation transformer voltage.

The procedure, as depicted in Figure 1, for predicting the nodes voltages of the HV side of the distribution network is as follows:

- We assumed that each terminal node on the distribution network is equipped with a smart meter to measure system parameters.

- We obtained the slack node voltage from the substation transformer.

- System operation data is acquired for active power $(P)$ and reactive power $(Q)$ of the nodes on the smart meter's distribution network.

- $\quad$ Our proposed scheme defines the HV-ML model to learn the mapping rules from the specified P, Q values to the acquired measurement data's node voltage to predict each node's voltage value.

Therefore, for a simplified two-bus topology as specified in Figure 2 with loads connected at the HV side of the distribution network with terminal voltages $V_{T}$, apparent power $S_{T}$, the junction impedance $Z_{J}$, and terminal impedance $Z_{T}$, could be assumed to be constant. The terminal voltage $V_{T}$, of the load could be expressed as a function of the 
junction voltage $V_{J 1}$, and the terminal's apparent power $S_{T}$. Hence, as depicted in (1) and (2), the correlation between voltage and power consumed by each load can be emphasized.

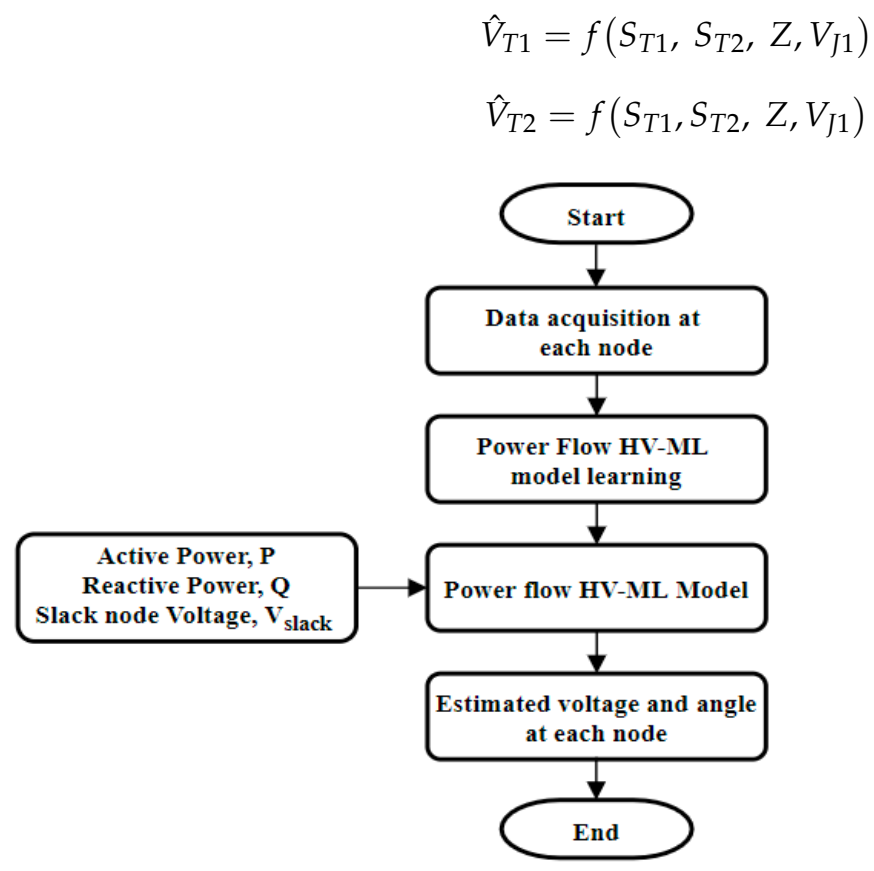

Figure 1. Flow chart of HV-ML Model.

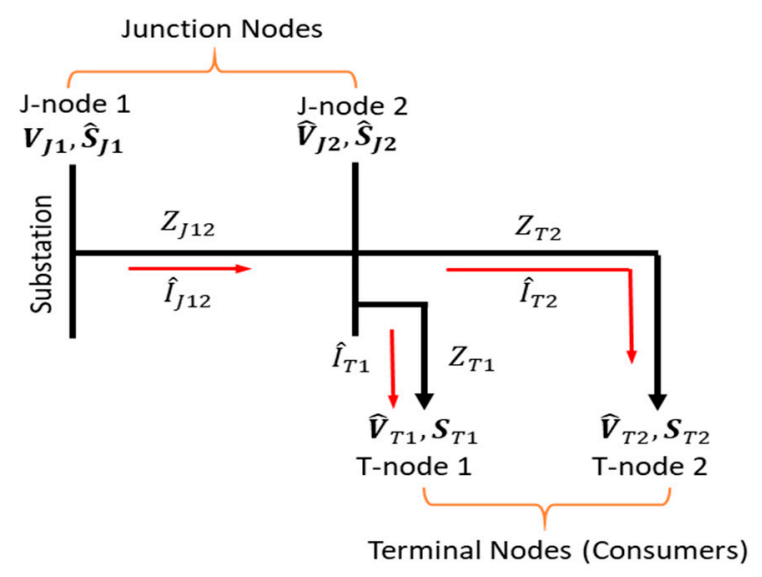

Figure 2. Simplified Linear High-Voltage Distribution network topology.

The structure of the HV-ML model is illustrated by way of a diagram in Figure 3. As shown in Table 1, it is a design based on three hidden layer multilayer perceptrons (MLPs). The number of neurons in the hidden layer needed to be adjusted. A suitable number of hidden neurons were determined by varying the number of hidden neurons. In the case study using this ML model, a structure containing three hidden layers of 15, 10, and 8 neurons between the input and out layers was adapted to realize the best performance.

As shown in Figure 3, for the HV-side of the distribution network, the terminal voltage can be predicted with the measurable junction voltage, $V_{J 1}$, and the terminals' apparent power, $S_{T}$. The efficacy of the terminal voltage, $V_{T}$, as the only predicted in such a model can be proven with the terminal node voltage equation of (1) and (2). The terminal node voltage equation expressed in terminal current, $\hat{I}_{T 1}$ in (3), could further be expressed in only measurable parameters in (4). The J-node voltage, $\hat{V}_{J 2}$ in (4) excludes the voltage drop occurring from the line impedance between the measured slack node voltage and the J-node as expressed in (5). 


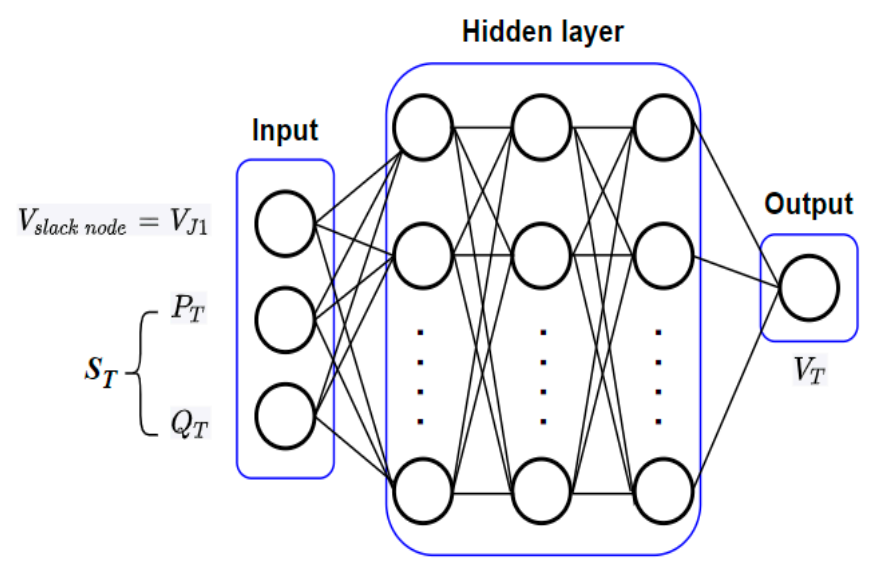

Figure 3. High-Voltage Machine Learning Model.

Table 1. Number of neurons for each layer in the structure of HV-ML.

\begin{tabular}{cc}
\hline ML Layer & No. of Neurons \\
\hline Input & 3 \\
Hidden 1 & 15 \\
Hidden 2 & 10 \\
Hidden 3 & 8 \\
Output & 1 \\
\hline
\end{tabular}

The J-node current, $\hat{I}_{J 12}$ in (5), is expressed in (6) in terms of the J-node voltage, $\hat{V}_{J 2}$, and apparent power, $\hat{S}_{J 2}$. The J-node current is further described in the network's measurable parameters: line voltage-drop, apparent power, and line impedance. Based on the network topology, $\hat{V}_{J 2}$ could be expressed in multiple ways; in this instance, $\hat{V}_{J 2}$ could be described in terms of T-node 1 or T-node 2 measurable parameters.

$$
\begin{gathered}
S_{T 1}=\hat{V}_{T 1} \hat{I}_{T 1}^{*} \\
S_{T 1}=\hat{V}_{T 1}\left(\frac{\hat{V}_{T 1}-\hat{V}_{J 2}}{Z_{T 1}}\right)^{*} \\
\hat{V}_{J 2}=V_{J 1}-Z_{J 12} \hat{I}_{J 12} \\
\hat{I}_{J 12}=\left(\frac{\hat{S}_{J 2}}{\hat{V}_{T 2}}\right)^{*}=\left(\frac{S_{T 1}+Z_{T 1}\left|\hat{I}_{T 1}\right|^{2}+S_{T 2}+Z_{T 2}\left|\hat{I}_{T 2}\right|^{2}}{Z_{T 2} \hat{I}_{T 2}+\hat{V}_{T 2}}\right)^{*} \\
=\left(\frac{S_{T 1}+Z_{T 1}\left|\hat{I}_{T 1}\right|^{2}+S_{T 2}+Z_{T 2}\left|\hat{I}_{T 2}\right|^{2}}{Z_{T 2} \hat{I}_{T 2}+\hat{V}_{T 2}}\right)^{*}
\end{gathered}
$$

Expressing (6) in (5) yields a quadratic equation in (7), the solution of which is the T-node voltage, $V_{T 1}$, as defined in (8).

$$
\begin{gathered}
S_{T 1} Z_{T 1}^{*}=\hat{V}_{T 1}\left(\hat{V}_{T 1}-V_{J 1}+Z_{J 12}\left(\frac{S_{T 1}+Z_{T 1}\left|\hat{I}_{T 1}\right|^{2}+S_{T 2}+Z_{T 2}\left|\hat{I}_{T 2}\right|^{2}}{Z_{T 2} \hat{I}_{T 2}+\hat{V}_{T 2}}\right)^{*}\right)^{*} \\
\hat{V}_{T 1}=\frac{\left[A \pm \sqrt{A^{2}-4 S_{T 1} Z_{T 1}^{*} B}\right]}{2 B}
\end{gathered}
$$

where:

$$
\begin{gathered}
A=V_{J 1}{ }^{*} B-Z_{J 12}\left(S_{T 1}+Z_{T 1}\left|\hat{I}_{T 1}\right|^{2}+S_{T 2}+Z_{T 2}\left|\hat{I}_{T 2}\right|^{2}\right) \\
B=Z_{T 2} \hat{I}_{T 2}+\hat{V}_{T 2}
\end{gathered}
$$




$$
\hat{I}_{T 1}=\sqrt{\frac{S_{T 1}}{Z_{T 1}}}, \hat{I}_{T 2}=\sqrt{\frac{S_{T 2}}{Z_{T 2}}}, \hat{V}_{T 2}=\frac{S_{T 2}}{\left(\sqrt{\frac{S_{T 2}}{Z_{T 2}}}\right)^{*}}
$$

Similarly, by deriving the equation for $V_{T 2}$, the voltage for T-node 2 can be verified as follows:

$$
\hat{V}_{T 2}=\frac{\left[C \pm \sqrt{C^{2}-4 S_{T 1} Z_{T 1}^{*} D}\right]}{2 D}
$$

where:

$$
\begin{gathered}
C=V_{J 1}{ }^{*} D-Z_{J 12}\left(S_{T 1}+Z_{T 1}\left|\hat{I}_{T 1}\right|^{2}+S_{T 2}+Z_{T 2}\left|\hat{I}_{T 2}\right|^{2}\right) \\
D=Z_{T 1} \hat{I}_{T 1}+\hat{V}_{T 1} \\
\hat{I}_{T 1}=\sqrt{\frac{S_{T 1}}{Z_{T 1}}}, \hat{V}_{T 1}=\frac{S_{T 1}}{\left(\sqrt{\frac{S_{T 1}}{Z_{T 1}}}{ }^{*}\right.}, \hat{I}_{T 2}=\sqrt{\frac{S_{T 2}}{Z_{T 2}}}
\end{gathered}
$$

Generally, multiple loads connect to the distribution network at the HV-side. As shown in Figure 4, a similar analysis is deduced to predict the network's terminal voltages for multiple loads. As mention earlier, for the HV distribution system, since the slack node is the substation transformer, the slack node voltage can be obtained. Hence, the various terminal voltages could be predicted with the model mentioned above. In such a situation, predicting the terminal loads' voltages is similar to the simplified model voltage prediction model described above. The generalized scheme to estimate the terminal loads' voltages is divided into Part A and Part B. For Part A, the number of terminal node voltages to estimate is more than two. The general process of predicting multiple terminal node voltages is detailly explained in Appendix A.

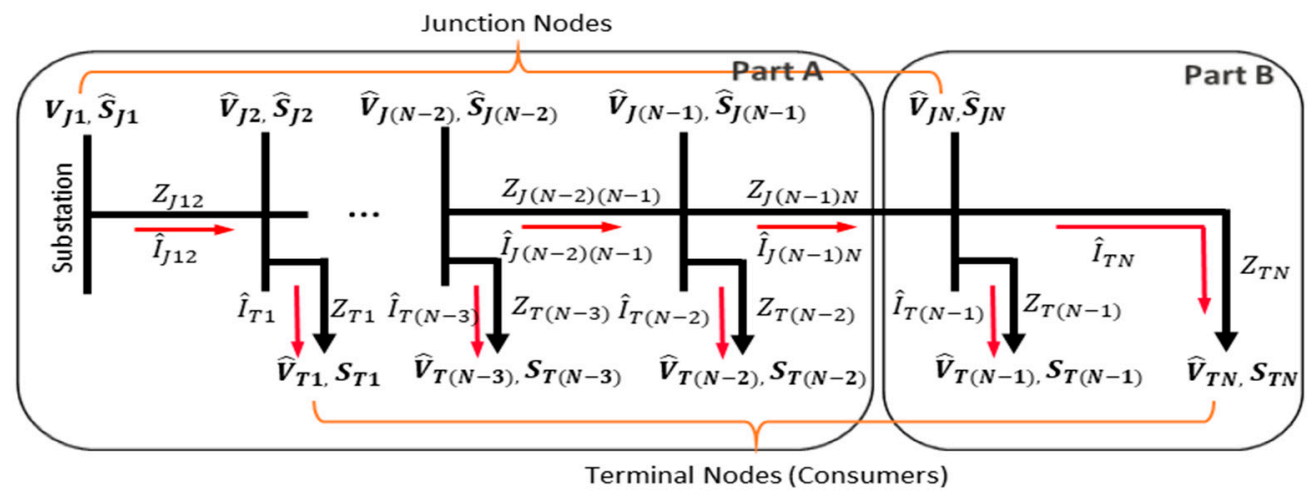

Figure 4. Generalized Linear High-Voltage Distribution network topology.

Part A:

$$
\begin{gathered}
\hat{V}_{T(N-i)}=\frac{E \pm \sqrt{E^{2}+4 S_{T(N-i)} Z_{T(N-i)}^{*}}}{2} \\
E=\left[V_{J 1}+\sum_{i=2}^{N-1} Z_{J(N-i),(N-(i-1)))} \hat{I}_{J(N-i),(N-(i-1)))}\right]^{*}
\end{gathered}
$$

Part B:

$$
\begin{aligned}
\hat{V}_{T(N-1)} & =\frac{F \pm \sqrt{F^{2}+4 S_{T(N-1)} Z_{T(N-1)}^{*}}}{2} \\
\hat{V}_{T(N)} & =\frac{G \pm \sqrt{G^{2}+4 S_{T(N)} Z_{T(N)}^{*}}}{2}
\end{aligned}
$$


where:

$$
F=G=\left[V_{J(1)}+\sum_{i=1}^{N-1} Z_{J(N-i),(N-(i-1)))} \hat{I}_{J(N-i),(N-(i-1)))}\right]^{*}
$$

\subsection{Low-Voltage Machine-Learning ( $L V-M L)$}

Contrary to the HV-ML model, the LV-ML model is time-dependent; the LV-ML Predicts the change in voltage, $\Delta V^{t}$, at a time, $t$, of each terminal node on the distribution network; with this, the terminal voltage, $V^{t+1}$, could be predicted.

For this, the LV-ML model is devised to predict the terminal voltage with unknown slack node voltage. The procedure, as depicted in Figure 5, for predicting the nodes voltages of the LV side of the distribution network is as follows:

- We assumed that each terminal time-dependent distribution network is equipped with a smart meter to measure system parameters.

- System operation data is acquired for the voltage, $\left(V^{t}\right)$, active power, $\left(P^{t}\right)$, reactive power, $\left(Q^{t}\right)$, and change in apparent power, $\left(\Delta S^{t}\right)$, of the nodes on the distribution network by the smart meter.

- Our proposed scheme defines the LV-ML model to learn the mapping rules from the specified $\mathrm{P}, \mathrm{Q}$ and change in apparent power values to the acquired measurement data's node change in voltage to predict each node's voltage value for a specified time.

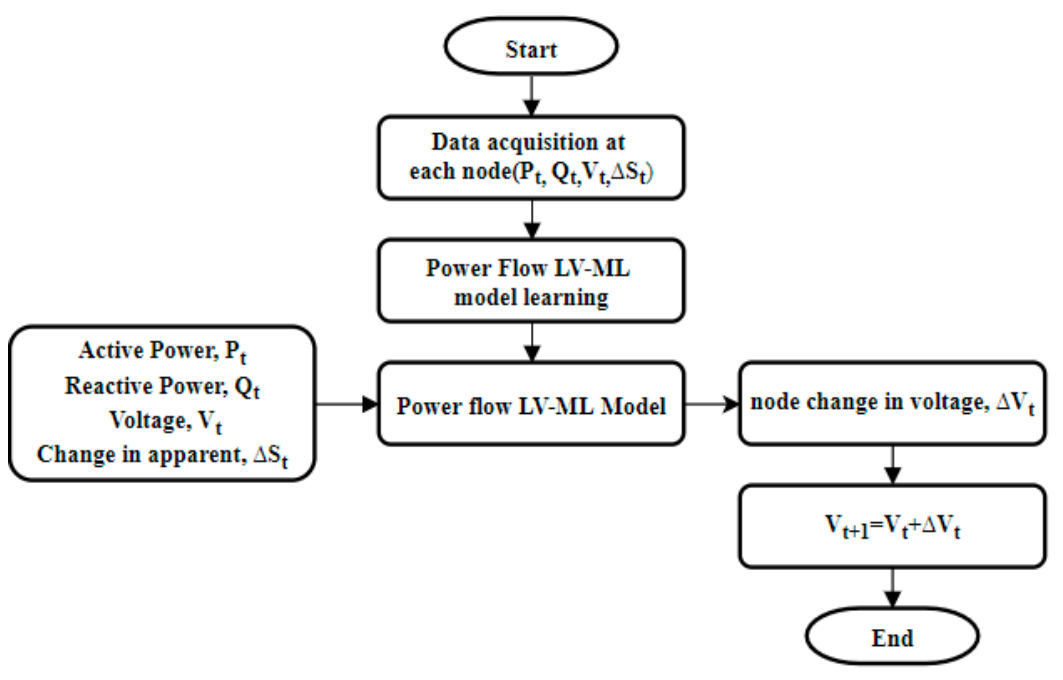

Figure 5. Flow chart of LV-ML Model.

Similarly, for the HV side of the distribution network, the terminal voltages of the LV side of the distribution network could be predicted. However, unlike the HV side of the distribution network, the distribution network's slack node is the pole transformer. The pole transformer voltage, $V_{J 1}$, at the J-node 1 , unlike the substation transformer, $V_{J 1}$ is not measurable. Hence the HV-ML model does not apply to the LV side of the distribution network. For a simplified two-bus topology as shown in Figure 6 with the loads connected at the LV side of the distribution network with terminal voltages $V^{t}$, and apparent power $S^{t}$, the junction impedance, $Z_{J}$, and terminal impedance, $Z_{T}$, could be assumed to be constant.

The voltage, $\hat{V}^{t+1}$, of the terminal load could be expressed in terms of its proceeding voltage, $V^{t}$, and the change in voltage, $\Delta \hat{V}^{t}$, as specified in (22) Similarly, the apparent power, $S^{t+1}$, could be expressed as defined in (21).

$$
\begin{aligned}
& S_{T}^{t+1}=S_{T}^{t}+\Delta S_{T}^{t} \\
& \hat{V}_{T}^{t+1}=V_{T}^{t}+\Delta \hat{V}_{T}^{t}
\end{aligned}
$$




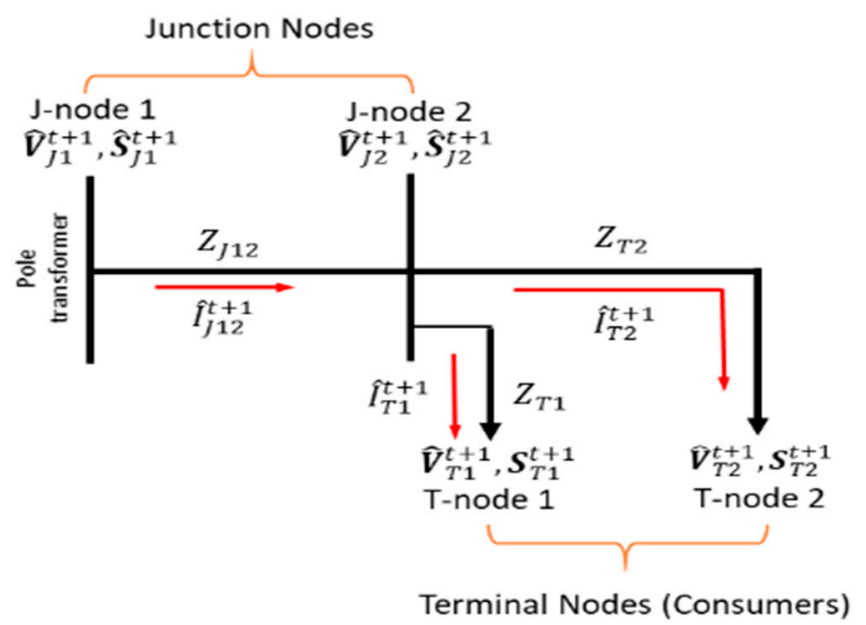

Figure 6. Simplified Linear Low-Voltage Distribution network topology.

The LV-ML model proposed in this section, contrary to the HV-ML model, predicts the amount of change in terminal voltage, $\Delta V_{T}^{t}$, with a change in apparent power, $\Delta S_{T}^{t}$, at a time, $\mathrm{t}$, of the terminal nodes as shown in Figure 7. Similar to the parameter measurement procedure mentioned earlier, the terminal voltages and apparent power are recorded simultaneously to train a model to predict a change in voltage. For this model, the terminal's apparent power, $S_{T}^{t}$, a change in apparent power, $\Delta S_{T}^{t}$, and voltage, $V_{T}^{t}$, serves as input with $\Delta V_{T}^{t}$ as the output to the model. The structure of the LV-ML model is illustrated by way of a diagram in Figure 7. As shown in Table 2, A suitable number of hidden neurons were determined by varying the number of hidden neurons. In the case study using this ML model, a structure containing three hidden layers of 20,15, and 10 neurons between the input and out layers were adapted to realize the best performance.

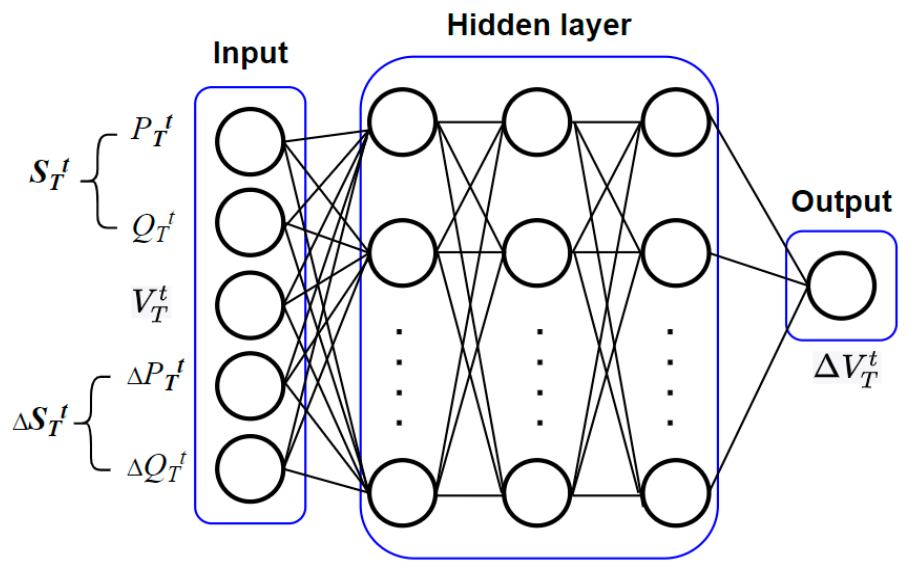

Figure 7. Low-Voltage Machine Learning Model.

Table 2. Number of neurons for each layer in the structure of LV-ML.

\begin{tabular}{cc}
\hline ML Layer & No. of Neurons \\
\hline Input & 5 \\
Hidden 1 & 20 \\
Hidden 2 & 15 \\
Hidden 3 & 10 \\
Output & 1 \\
\hline
\end{tabular}

The change in the terminal voltage, $\Delta V_{T}$ at the terminal node could be expressed as a function of the terminal's apparent power, $S_{T}$, Voltage, $V_{T}$, change in apparent power, 
$\Delta S_{T}$, and line impedance, $Z$. Hence, as expressed in (23) and (24), the correlation between voltage and power consumed by each load can be emphasized.

$$
\begin{aligned}
& \Delta \hat{V}_{T 1}^{t}=f\left(S_{T 1}^{t}, S_{T 2}^{t}, Z, V_{T 1}^{t}, V_{T 2}^{t}, \Delta S_{T 1}^{t}, \Delta S_{T 2}^{t}\right) \\
& \Delta \hat{V}_{T 2}^{t}=f\left(S_{T 1}^{t}, S_{T 2}^{t}, Z, V_{T 1}^{t}, V_{T 2}^{t}, \Delta S_{T 1}^{t}, \Delta S_{T 2}^{t}\right)
\end{aligned}
$$

To prove that the amount of voltage change can be predicted according to the measurable parameters and the change in power, $\Delta S_{T}$, and voltage, $\Delta V_{T}$, equation of the T-node is verified with the simplified network topology in Figure 6. As shown in the figure, the network consists of measurable parameters: apparent power, $S_{T}^{t}$, and the change in apparent power, $\Delta S_{T}^{t}$, and voltage, $V_{T}^{t}$. Contrary to the HV model, the initial slack node voltage of the LV side of the distribution network is predicted under this model.

$$
V_{J 1}^{t}=V_{J 2}^{t}+Z_{J 12} I_{J 12}^{t} \cong \hat{V}_{J 1}^{t+1}
$$

For a slight change in time, $\Delta t$, the voltage fluctuation of the slack node is negligible. Therefore, for this model, the slack node voltage, $V_{J 1}^{t+1}$ at J-node 1 , is assumed to be the same as $V_{J 1}^{t}$ for a slight change in time, $\Delta t$, as shown in (25). The proposed model is not adaptable to situations with much variance in the slack node voltage for time $t$ and $t+1$. As shown in Figure 8, there is considerable variance in the predicted voltage change with many voltage variations between successive periods.

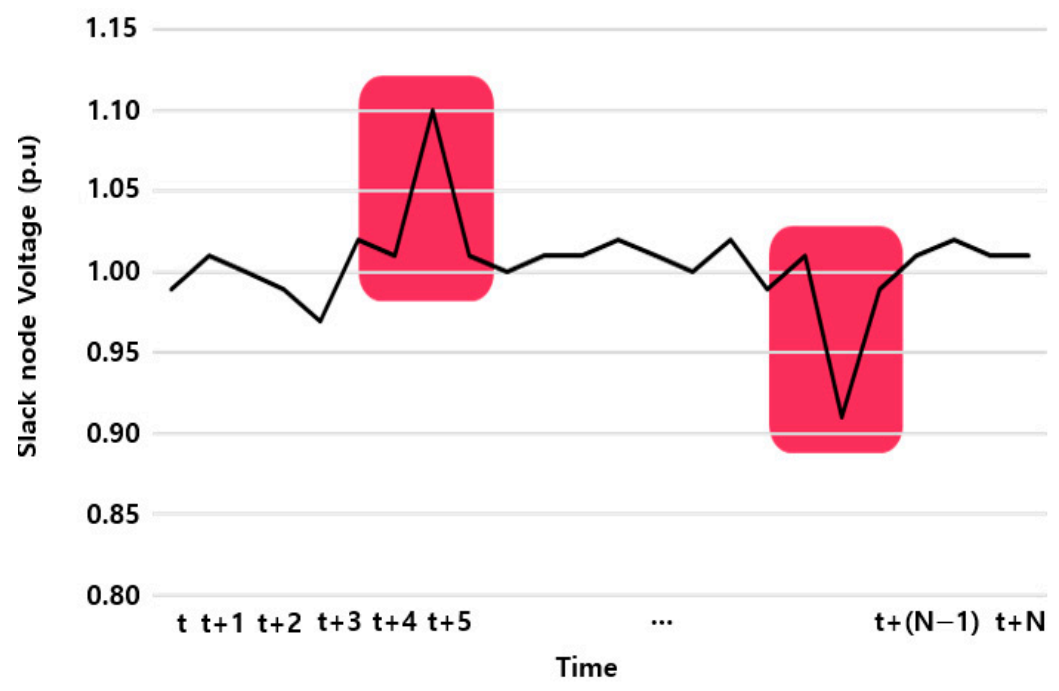

Figure 8. Special Event of slack node voltage.

In Figure 6, the power equation of T-node 1 at the time $t+1$ defined in (21) can be expressed in terms of current, $I_{T 1}^{t+1}$, and voltage, $V_{T 1}^{t+1}$, as in (26) J-node current, $I_{J 12}^{t+1}$ in (27), is expressed in terms of power, $S_{J 2}^{t+1}$, J-node voltage, $V_{J 2}^{t+1}$, and the line impedance in (29). Similar to the HV model, the J-node voltage, $V_{I 2}^{t+1}$, can be expressed in two ways: changing the equation according to the voltage change amount on the terminal node to be calculated.

$$
\begin{gathered}
S_{T 1}^{t+1}=\hat{V}_{T 1}^{t+1} * \hat{I}_{T 1}^{t+1 *} \\
S_{T 1}^{t+1}=\hat{V}_{T 1}^{t+1} *\left(\frac{\hat{V}_{T 1}^{t+1}-\hat{V}_{J 2}^{t+1}}{Z_{T 1}}\right)^{*} \\
\hat{V}_{J 2}^{t+1}=V_{J 1}^{t+1}-Z_{J 12} \hat{I}_{J 12}^{t+1}
\end{gathered}
$$




$$
\begin{gathered}
\hat{I}_{J 12}^{t+1}=\left(\frac{\hat{S}_{I 2}^{t+1}}{\hat{V}_{T 2}^{t+1}}\right)^{*}=\left(\frac{S_{T 1}^{t+1}+\left.Z_{T 1}||_{T 1}^{t+1}\right|^{2}+S_{T 2}^{t+1}+Z_{T 2}\left|\hat{I}_{T 2}^{t+1}\right|^{2}}{Z_{T 2} \hat{I}_{T 2}^{t+1}+\hat{V}_{T 2}^{t+1}}\right)^{*} \\
=\left(\frac{S_{T 1}^{t+1}+Z_{T 1}\left|\hat{I}_{T 1}^{t+1}\right|^{2}+S_{T 2}^{t+1}+Z_{T 2}\left|I_{T 2}^{t+1}\right|^{2}}{Z_{T 1} \hat{I}_{T 1}^{t+1}+\hat{V}_{T 1}^{t+1}}\right)^{*} \\
S_{T 1}^{t+1} Z_{T 1}^{*}=\hat{V}_{T 1}^{t+1}\left(\hat{V}_{T 1}^{t+1}-V_{J 1}^{t+1}+Z_{J 12}\left(\frac{S_{T 1}^{t+1}+Z_{T 1}\left|\hat{I}_{T 1}^{t+1}\right|^{2}+S_{T 2}^{t+1}+Z_{T 2}\left|\hat{I}_{T 2}^{t+1}\right|^{2}}{Z_{T 2} \hat{I}_{T 2}^{t+1}+\hat{V}_{T 2}^{t+1}}\right)^{*}\right)^{*}
\end{gathered}
$$

Similar to the HV model, expressing (29) in (28) yields a quadratic equation in (30), the solution of which is the T-node 1 voltage change, $\Delta V_{T 1}^{t}$ and the T-node 2 voltage change, $\Delta V_{T 2}^{t}$ as expressed in (31) and (35).

$$
\Delta \hat{V}_{T 1}^{t}=\frac{H \pm \sqrt{H^{2}+4 S_{T 1}^{t+1} Z_{T 1}^{*} I^{2}}}{2 I}-V_{T 1}^{t}
$$

where:

$$
\begin{gathered}
H=\left(Z_{T 2} \hat{I}_{T 2}^{t+1}+\hat{V}_{T 2}^{t+1}\right)\left(V_{J 2}^{t}+Z_{J 12} I_{J 12}^{t}\right)^{*}+Z_{J 12}\left(S_{T 1}^{t+1}+Z_{T 1}\left|\hat{I}_{T 1}^{t+1}\right|^{2}+S_{T 2}^{t+1}+Z_{T 2}\left|\hat{I}_{T 2}^{t+1}\right|^{2}\right) \\
K=\left(Z_{T 2} \hat{I}_{T 2}^{t+1}+\hat{V}_{T 2}^{t+1}\right) \\
\hat{I}_{T 1}^{t+1}=\sqrt{\frac{S_{T 1}^{t+1}}{Z_{T 1}}}, \hat{I}_{T 2}^{t+1}=\sqrt{\frac{S_{T 2}^{t+1}}{Z_{T 2}}, \hat{V}_{T 2}^{t+1}=\frac{S_{T 2}^{t+1}}{\left(\sqrt{\frac{S_{T 2}^{t+1}}{Z_{T 2}}}\right)}} \\
\Delta \hat{V}_{T 2}^{t}=\frac{J \pm \sqrt{J^{2}+4 S_{T 1}^{t+1} Z_{T 1}^{*} I^{2}}}{2 K}-V_{T 2}^{t}
\end{gathered}
$$

where:

$$
\begin{gathered}
J=\left(Z_{T 1} \hat{I}_{T 1}^{t+1}+\hat{V}_{T 1}^{t+1}\right)\left(V_{J 2}^{t}+Z_{J 12} I_{J 12}^{t}\right)^{*}+Z_{J 12}\left(S_{T 1}^{t+1}+Z_{T 1}\left|\hat{I}_{T 1}^{t+1}\right|^{2}+S_{T 2}^{t+1}+Z_{T 2}\left|\hat{I}_{T 2}^{t+1}\right|^{2}\right) \\
K=\left(Z_{T 1} \hat{I}_{T 1}^{t+1}+\hat{V}_{T 1}^{t+1}\right) \\
\hat{I}_{T 1}^{t+1}=\sqrt{\frac{S_{T 1}^{t+1}}{Z_{T 1}}}, \hat{I}_{T 2}^{t+1}=\sqrt{\frac{S_{T 2}^{t+1}}{Z_{T 2}}}, \hat{V}_{T 1}^{t+1}=\frac{S_{T 1}^{t+1}}{\left(\sqrt{\frac{S_{T 2}^{t+1}}{Z_{T 1}}}\right)}
\end{gathered}
$$

Like the high-voltage system, the voltage prediction model that can be used when there are two loads in the distribution system, as shown in Figure 9, has been proved, and the generalized equation for the case where there are several loads is proved.

The general process of predicting multiple terminal node voltages is detailly explained in Appendix A. It is generalized to predict the voltage according to the amount of power change on the load side, and it is organized by dividing the load side of the low voltage distribution system into Part A and Part B like the high-voltage distribution system. For Part A, (39) and Part B is defined as (41)

Part A:

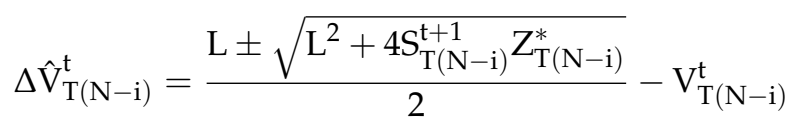

where:

$$
\mathrm{L}=\left[\mathrm{V}_{\mathrm{J} 2}^{\mathrm{t}}+\mathrm{Z}_{\mathrm{J} 12} \mathrm{I}_{\mathrm{J} 12}^{\mathrm{t}}+\sum_{\mathrm{i}=2}^{\mathrm{N}-1} \mathrm{Z}_{\mathrm{J}(\mathrm{N}-(\mathrm{i}-1), \mathrm{N}-\mathrm{i}))} \hat{\mathrm{I}}_{\mathrm{J}(\mathrm{N}-(\mathrm{i}-1), \mathrm{N}-\mathrm{i}))}^{\mathrm{t}+1}\right]^{*}
$$




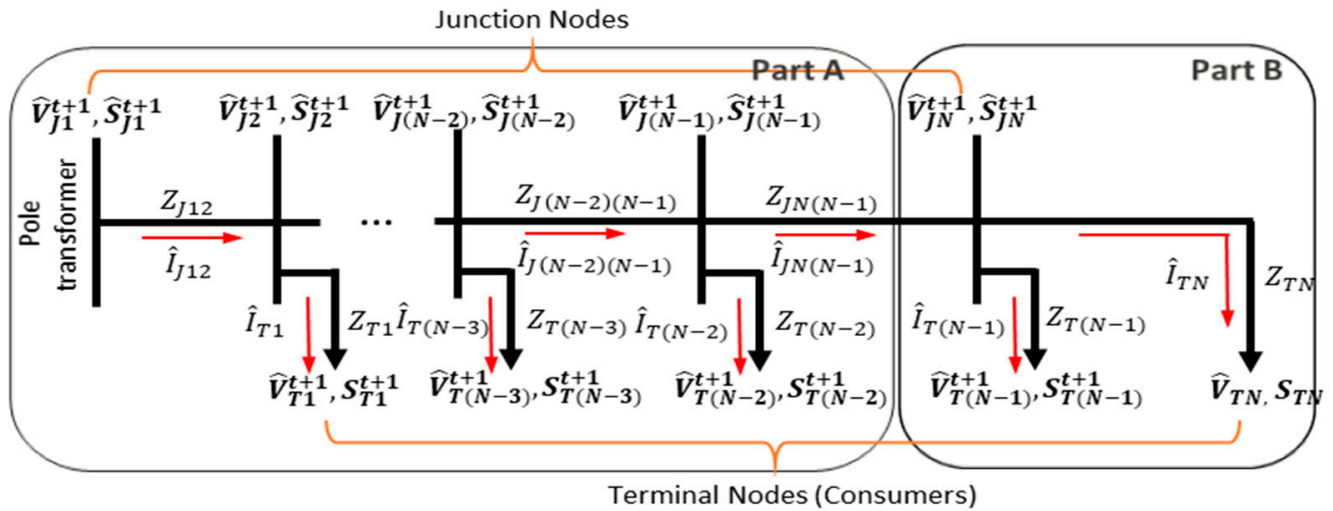

Figure 9. Generalized Linear Low-Voltage Distribution network topology.

Part B:

$$
\begin{gathered}
\Delta \hat{\mathrm{V}}_{\mathrm{T}(\mathrm{N}-1)}=\frac{\mathrm{M} \pm \sqrt{\mathrm{M}^{2}+4 \mathrm{~S}_{\mathrm{T}(\mathrm{N}-\mathrm{i})}^{\mathrm{t}+1} \mathrm{Z}_{\mathrm{T}(\mathrm{N}-1)}^{*}}}{2}-\mathrm{V}_{\mathrm{T}(\mathrm{N}-1)}^{\mathrm{t}} \\
\Delta \hat{\mathrm{V}}_{\mathrm{T}(\mathrm{N})}=\frac{\mathrm{N} \pm \sqrt{\mathrm{N}^{2}+4 \mathrm{~S}_{\mathrm{T}(\mathrm{N})}^{\mathrm{t}+1} \mathrm{Z}_{\mathrm{T}(\mathrm{N})}^{*}}-\mathrm{V}_{\mathrm{T}(\mathrm{N})}^{\mathrm{t}}}{2}
\end{gathered}
$$

where:

$$
\mathrm{M}=\mathrm{N}=\left[\mathrm{V}_{\mathrm{J} 2}^{\mathrm{t}}+\mathrm{Z}_{\mathrm{J} 12} \mathrm{I}_{\mathrm{J} 12}^{\mathrm{t}}+\sum_{\mathrm{i}=1}^{\mathrm{N}-1} \mathrm{Z}_{\mathrm{J}(\mathrm{N}-\mathrm{i}),(\mathrm{N}-(\mathrm{i}-1)))} \hat{\mathrm{I}}_{\mathrm{J}(\mathrm{N}-(\mathrm{i}-1), \mathrm{N}-\mathrm{i}))}^{\mathrm{t}+1}\right]^{*}
$$

\section{Case Study}

To evaluate our proposed models' efficacy, we performed a series of experiments in a realistic environment. Adopting IEEE 33-bus topology as depicted in Figure 10; thirty-two nodes were designated terminal nodes (T-node) and twenty-nine junction nodes (J-node). The first junction node was connected to the transformer, and the remaining nodes were equally paced on the distribution network. At each T-node, a smart meter was installed to measure the terminal power. To construct the sample data, the active power and reactive power measured at each T-node are collected, and the power flow is performed using the collected data. The obtained voltage $(V)$, active power $(P)$, and reactive power $(Q)$ are used as learning data.

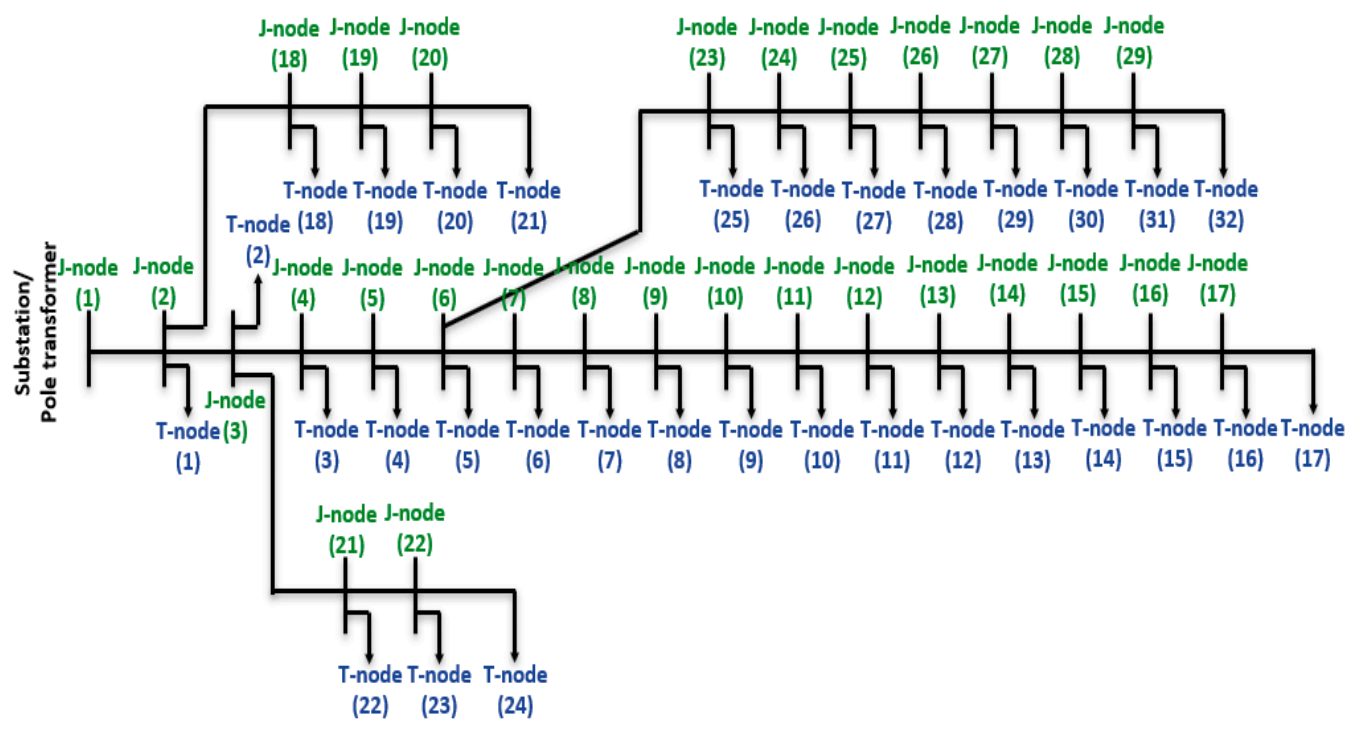

Figure 10. A typical IEEE 33-bus distribution network topology. 
A different set of experiments were designed for the HV and LV models to apply our proposed models for voltage prediction. However, the same set of measured parameters was used in each case.

The experiment setup measured the voltage and power of all nodes within hourly intervals for 60 days. A total of 1440 data samples were generated for each measured parameter for each node. Figure 11 shows a $24 \mathrm{~h}$ load profile of active power, reactive power, and voltage of the nodes on the distribution network. Training and validation data were set to $80 \%$ and $20 \%$ of total data samples and the test set used 24 data that were not used for training and validation. The procedure for evaluating the effectiveness of the proposed models are as follows:

- $\quad$ The HV-ML model learns a mapping relationship between the measured terminal node's apparent power and slack node voltage as input parameters and terminal node voltage as an output parameter. The trained model is subsequently used to predict the terminal voltage with a given set of apparent power and slack node voltage. An error rate is predicted based on the measured and predicted voltage values to show the proposed model's performance.

- However, in LV voltage prediction, the measured terminal nodes' apparent power, voltage, and current are used to estimate the line impedances. With an assumed change in apparent power and the estimated line impedances, the power flow analysis is performed to predict the change in voltage. Similarly, with the assumed change in apparent power and other measurable parameters, the LV-ML model predicts the terminal voltage change for all nodes. An error rate is predicted for both the power flow analysis method and the LV-ML model. The error rate shows the performance of the proposed model against the benchmark. Contrary to the HV-ML performance evaluation, each node's LV-ML voltage prediction is done for each hour. The minimum, maximum, and average daily error rate is predicted.

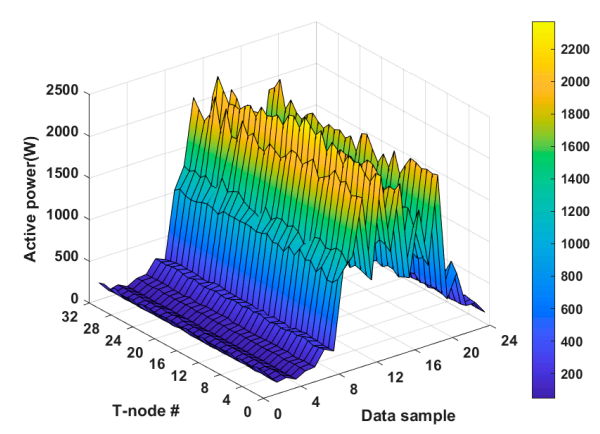

(a) T-nodes active power

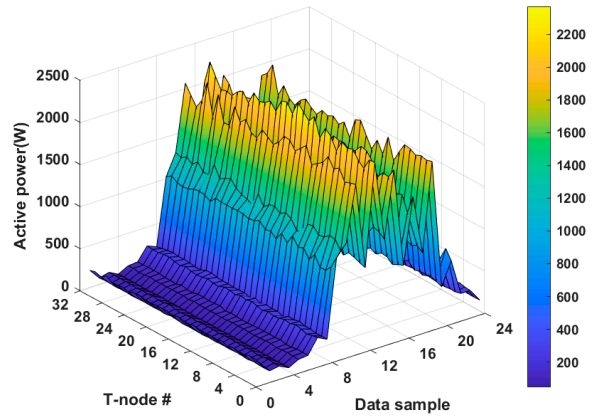

(b) T-nodes reactive power

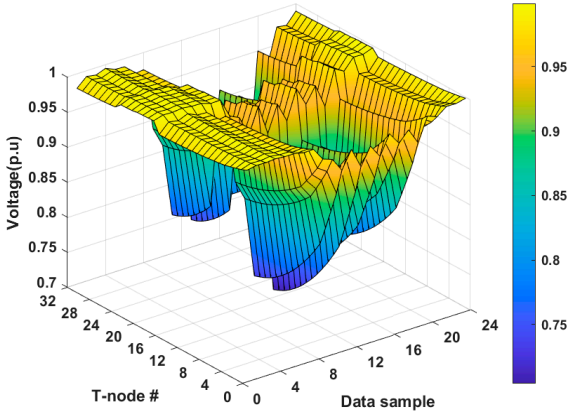

(c) T-nodes voltage

Figure 11. T-nodes Load profile.

\subsection{Case I: Effect of Data Sample Size on High-Voltage Prediction}

The HV-ML model predicts the terminal node voltages on the high side of the distribution network. A measured set of apparent power and voltage of the terminal nodes were used to train the HV-ML model. The apparent power and the slack node voltage were input parameters to the defined model, whereas the terminal node voltage was the output parameter of the model. To predict the minimum sample data size required for the HV model's efficient running, the performance of the HV-ML model was evaluated on different training and validation data samples: 10, 50, 100, 300, 500, 1000, and 1440. These different data samples were used to train HV-ML models for high voltage prediction. The results of the model's predictions, as expressed as error rates for the various terminal nodes and the average error rate for each sample data, are shown in Table 3. 
Table 3. T-node voltage prediction error rate (\%) at 1.0 (p.u) slack node voltage.

\begin{tabular}{|c|c|c|c|c|c|c|c|}
\hline $\begin{array}{c}\text { \# of the Samples } \\
\text { T-Node }\end{array}$ & 10 & 50 & 100 & 300 & 500 & 1000 & 1440 \\
\hline 1 & 1.192 & 1.025 & 0.942 & 0.871 & 0.011 & 0.01 & 0.01 \\
\hline 2 & 2.202 & 1.922 & 1.746 & 1.62 & 0.004 & 0.004 & 0.004 \\
\hline 3 & 3.146 & 2.79 & 2.518 & 2.362 & 0.013 & 0.011 & 0.011 \\
\hline 4 & 4.074 & 3.618 & 3.288 & 3.085 & 0.02 & 0.02 & 0.02 \\
\hline 5 & 4.984 & 4.431 & 4.032 & 3.789 & 0.041 & 0.042 & 0.042 \\
\hline 6 & 5.501 & 4.914 & 4.46 & 4.168 & 0.056 & 0.055 & 0.055 \\
\hline 7 & 5.979 & 5.358 & 4.897 & 4.521 & 0.07 & 0.07 & 0.07 \\
\hline 8 & 6.398 & 5.763 & 5.281 & 4.844 & 0.036 & 0.036 & 0.036 \\
\hline 9 & 6.79 & 6.119 & 5.62 & 5.119 & 0.049 & 0.047 & 0.047 \\
\hline 10 & 7.155 & 6.432 & 5.948 & 5.378 & 0.046 & 0.047 & 0.047 \\
\hline 11 & 7.458 & 6.71 & 6.203 & 5.61 & 0.046 & 0.045 & 0.045 \\
\hline 12 & 7.742 & 6.98 & 6.448 & 5.801 & 0.06 & 0.06 & 0.06 \\
\hline 13 & 7.973 & 7.171 & 6.634 & 5.954 & 0.053 & 0.053 & 0.053 \\
\hline 14 & 8.158 & 7.332 & 6.777 & 6.078 & 0.081 & 0.081 & 0.081 \\
\hline 15 & 8.299 & 7.427 & 6.879 & 6.161 & 0.052 & 0.052 & 0.052 \\
\hline 16 & 8.369 & 7.499 & 6.946 & 6.21 & 0.06 & 0.06 & 0.06 \\
\hline 17 & 8.412 & 7.539 & 6.939 & 6.225 & 0.049 & 0.048 & 0.048 \\
\hline 18 & 1.516 & 1.26 & 1.074 & 0.951 & 0.01 & 0.0099 & 0.0099 \\
\hline 19 & 1.602 & 1.317 & 1.103 & 0.982 & 0.008 & 0.008 & 0.008 \\
\hline 20 & 1.667 & 1.366 & 1.134 & 0.996 & 0.013 & 0.013 & 0.013 \\
\hline 21 & 1.675 & 1.358 & 1.125 & 0.99 & 0.016 & 0.015 & 0.015 \\
\hline 22 & 2.424 & 2.115 & 1.916 & 1.751 & 0.022 & 0.021 & 0.021 \\
\hline 23 & 2.458 & 2.155 & 1.955 & 1.773 & 0.01 & 0.01 & 0.01 \\
\hline 24 & 2.464 & 2.16 & 1.941 & 1.766 & 0.007 & 0.007 & 0.007 \\
\hline 25 & 6.125 & 5.355 & 4.87 & 4.701 & 0.045 & 0.045 & 0.045 \\
\hline 26 & 6.455 & 5.646 & 5.128 & 4.962 & 0.049 & 0.050 & 0.050 \\
\hline 27 & 6.74 & 5.87 & 5.327 & 5.196 & 0.054 & 0.054 & 0.054 \\
\hline 28 & 6.986 & 6.057 & 5.497 & 5.401 & 0.069 & 0.068 & 0.068 \\
\hline 29 & 7.192 & 6.183 & 5.631 & 5.566 & 0.078 & 0.077 & 0.077 \\
\hline 30 & 7.367 & 6.299 & 5.715 & 5.694 & 0.093 & 0.092 & 0.092 \\
\hline 31 & 7.472 & 6.337 & 5.783 & 5.779 & 0.078 & 0.078 & 0.078 \\
\hline 32 & 7.549 & 6.362 & 5.845 & 5.857 & 0.041 & 0.041 & 0.041 \\
\hline Average & 5.422 & 4.777 & 4.362 & 4.067 & 0.0418 & 0.0415 & 0.0415 \\
\hline
\end{tabular}

To understand the trend in each data sample's prediction accuracy, the average error rate of all the t-node voltage predictions for each data sample is depicted in Figure 12. From this figure, there is a general trend with a larger sampling size. The error rate is inversely proportional to the data sample size. As shown in Figure 12, the error rate decreases with increasing data sample size. However, as the training and validation sample size reaches 500, the error rate saturates. A further increase in the data sample size does not cause a significant decrease in the error rate. Therefore, for better performance, with less computational time and minimized computational resources, the terminal voltages of the distribution network could be predicted with the HV-ML model with a sample-training data sample.

\subsection{Case II: Effect of Data Slack Node Voltage on Low-Voltage Prediction}

This case study investigates the effect of slack node voltage on the terminal node voltage prediction. Contrary to the HV-ML model, the LV-ML model predicts the terminal node voltages on the distribution network's low voltage side. Like the HV-ML model definition, a set of measured system parameters defines the LV-ML model for each distribution network. However, unlike the HV-ML model, this model assumes an unknown slack node voltage. Hence, the slack node voltage is not considered as an input parameter. With a supposed terminal node change in apparent power, apparent power, and voltage as input parameters and terminal node change in voltage as output parameter, the LV-ML is defined 
with a training data sample set. Proceeding this model definition, the line impedances are estimated based on the measured terminal nodes' apparent power, voltage, and current. With the estimated line impedances, the power flow analysis is also performed on all nodes on the distribution network.

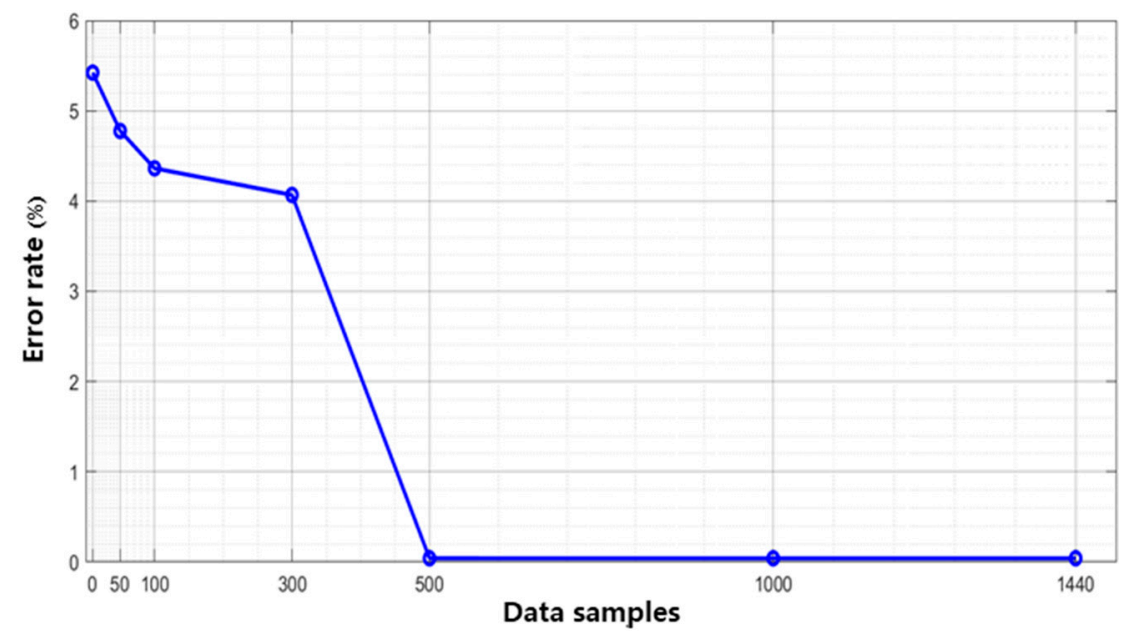

Figure 12. The saturation point of the HV-ML model was obtained by using sample data.

Like case study I, the experiment is repeated for different training and validation data samples: 10, 50, 100, 300, and 500 for a specified slack node voltage. This is to predict the minimum sample data size required for better prediction performance. The reason for limiting the number of samples to 500 in the LV-ML model is that a minimum of 500 samples is required to learn the HV-ML model as mentioned in the previous chapter. Therefore, we set the maximum number of samples for the LV-ML model to 500 and find out the minimum number of samples for the LV-ML case. These different data samples were used to train LV-ML models for the t-nodes change in voltage predictions. Unlike the high voltage prediction model, the low voltage prediction model is a function of time. Therefore, the T-node change in voltage is predicted hourly for each node in a 24-h window. The results of the prediction are as a root-mean-square error (RMSE). The daily minimum and maximum RMSE values for each prediction are then recorded. With an assumed slack node of 0.97 (p.u), the experiment is performed for the different training data samples.

To understand the trend in each training data sample's prediction accuracy, the RMSE value of all the t-node voltage predictions for each training data sample is depicted in Figure 13. Similar to the HV-ML model, the figure shows a decrease in RMSE value with increased training data sample size for the proposed model. However, the same is not the case with the impedance estimation method. The poor performance of the impedance estimation method is since the model assumes a constant 1.0 (p.u) slack node voltage. Hence, even with an increased training data sample, the model fails to adjust to preset slack node voltage. So, in such a case, it is evident that our proposed model has a better performance when compared with the impedance estimation method. Like the previous case study, there is a general trend with our model prediction. Our proposed model RMSE values are inversely proportional to the training data sample size. The RMSE decreases with increasing training data sample size. However, as the training data sample size reaches 300, the error rate saturates. A further increase in the training data sample size does not cause a significant decrease in the error rate. Therefore, for better performance, with less computational time and minimized computational resources, the terminal nodes' low voltages of the distribution network could be predicted with the LV-ML model with a sample training data sample. The low voltage prediction experiment is further repeated for different slack nodes: 1.0 (p.u) and 1.03 (p.u). The results of the experiment with 1.0 (p.u) slack node are presented in Figure 14. In contrast, the experiment results with 1.03 (p.u) slack node are shown in Figure 15. 


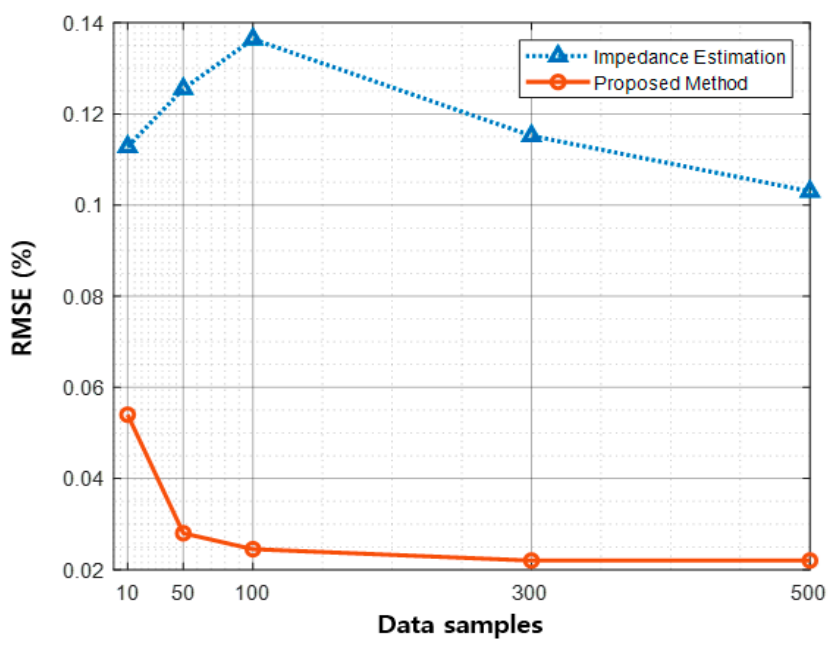

Figure 13. Effect of the data sample on the voltage prediction at 0.97 (p.u) slack node voltage.

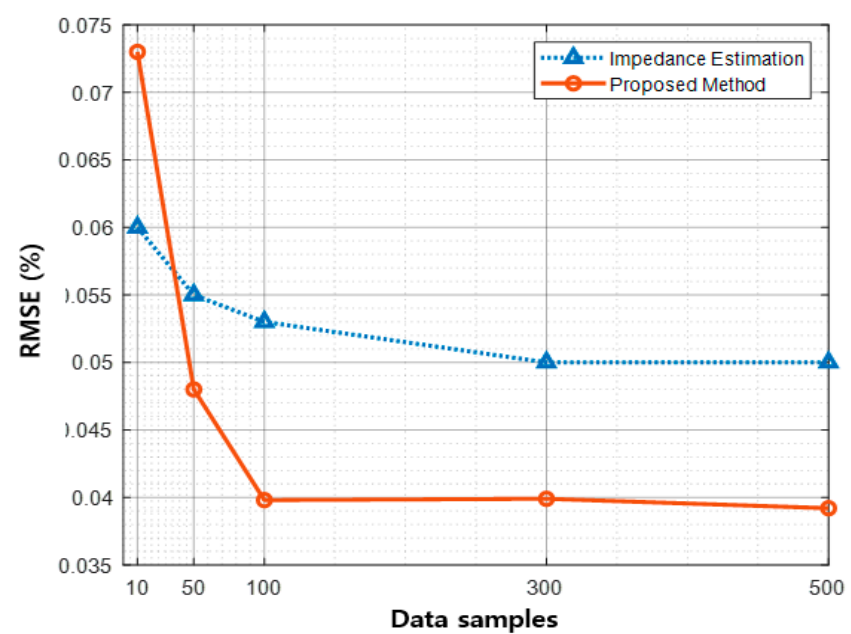

Figure 14. Effect of the data sample on the voltage prediction at 1.0 (p.u) slack node voltage.

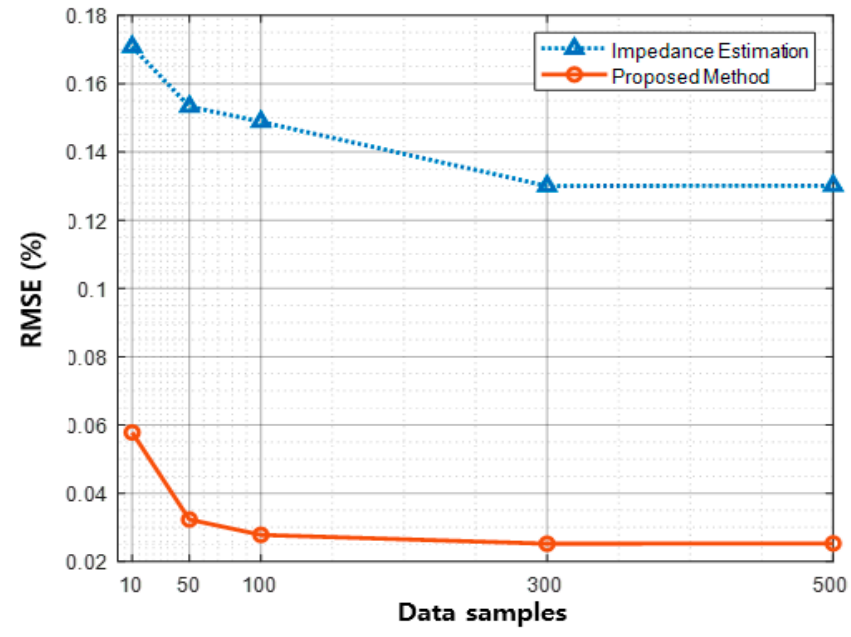

Figure 15. Effect of the data sample on the voltage prediction at 1.03 (p.u) slack node voltage.

The impedance estimation method performs better than our proposed model from Figure 14, even though it has smaller data samples. There is a steep in our proposed model's performance level when the data sample is 50. This is because much data is required for the neural network to capture a significant number of variabilities in the dataset. Hence, 
our proposed method continuously performs better than the impedance method when increasing the training data sample size. However, the performance of our proposed model saturates when the data sample is 100 . A further increase in the training data sample size does not cause a significant decrease in the error rate.

Similar to the 0.97 (p.u) slack node T-node voltage prediction experiment, the trend in our proposed model's performance results as predicted. The impedance estimation method was vast when the slack node voltage was 1.03 (p.u), as depicted in Figure 15. Generally, there is a decrease in the RMSE value from the figure with the increase in the training data sample size for both prediction methods. The poor performance of the impedance estimation method is because the model assumes a constant 1.0 (p.u) slack node voltage. Hence, even with an increased training data sample, the model fails to adjust to preset slack node voltage. So, in such a case, it is evident that our proposed model has a better performance when compared with the impedance estimation method. Like the previous case study, there is a general trend with our model prediction. Both models' RMSE values are inversely proportional to the training data sample size. The RMSE decreases with increasing training data sample size. However, our proposed model reaches an earlier saturation point than the impedance estimation method. A further increase in the training data sample size does not cause a significant decrease in the error rate. Therefore, for better performance, with less computational time and minimum computational resources, the terminal nodes' low voltages of the distribution network could be predicted with the LV-ML model with sample training data.

\section{Conclusions}

In this paper, we proposed a novel method that predicts the node voltages of the high-voltage and low-voltage sides of the distribution network. The voltage predictions are performed using practical measurable parameters. The models presented predicts terminal node voltages for the High and Low-side of the distribution network without the knowledge of topology and line impedances. The experiment results of the predicted voltages verify the performance and superiority of the proposed model relative to the conventional impedance estimation power flow analysis. In addition, the proposed method was used with the various load. The simulation results confirmed that the proposed method could estimate the voltage of T-nodes regardless of topology and line impedances.

The proposed method contributes to the analysis of the complex distribution system. Therefore, the proposed model can be extended to other reliability and stability analysis on the distribution system. In future work, other deep learning models, such as a recurrent neural network (RNN) and long short-term memory (LSTM) neural network, can be used in the model to enhance its performance.

Author Contributions: Conceptualization, J.P. and S.H.; methodology, J.P.; software, J.P.; validation, D.K. and K.A.A.; formal analysis, J.P., D.K., K.A.A. and T.J.; investigation, D.K., K.A.A. and T.J.; resources, J.P.; data curation, J.P., D.K., K.A.A. and T.J.; writing-original draft preparation, J.P.; writing-review and editing, J.P.; visualization, J.P.; supervision, S.H.; project administration, S.H.; funding acquisition, S.H. All authors have read and agreed to the published version of the manuscript.

Funding: This work is supported by the Korea Agency for Infrastructure Technology Advancement (KAIA) grant funded by the Ministry of Land, Infrastructure and Transport (grant number: 21HSCTB157917-02).

Institutional Review Board Statement: Not applicable.

Informed Consent Statement: Not applicable.

Data Availability Statement: Not applicable.

Conflicts of Interest: The authors declare no conflict of interest. 


\section{Appendix A}

Multiple Load model prediction. This section describes the derivation process of the generalized (16). The power relation of the T-node to be predicted can be derived as follows. Here, $I_{T(N-i)}^{*}$, can be expressed as (A1) again.

$$
\begin{gathered}
S_{T(N-i)}=\hat{V}_{T(N-i)} * \hat{I}_{T(N-i)}^{*} \\
S_{T(N-i)}=\hat{V}_{T(N-i)} *\left(\frac{\hat{V}_{T(N-i)}-\hat{V}_{J(N-(i-1))}}{Z_{T(N-i)}}\right)^{*} \\
S_{T(N-i)} Z_{T(T-i)}^{*}=\hat{V}_{T(N-i)}\left(\hat{V}_{T(N-i)}-\hat{V}_{J(N-(i-1))}\right)^{*} \\
\left|\hat{V}_{T(N-i)}\right|^{2}-\hat{V}_{T(N-i)} \hat{V}_{J(N-(i-1))}^{*}-S_{T(N-i)} Z_{T(T-i)}^{*}=0 \\
\hat{V}_{T(N-i)}=\frac{\hat{V}_{J(N-(i-1))}^{*} \pm \sqrt{\hat{V}_{J(N-(i-1))}^{2 *}-4 S_{T(N-i)} Z_{T(T-i)}^{*}}}{2} \\
\hat{V}_{J(N-(i-1))}=V_{J 1}+\sum_{i=2}^{N-1} Z_{J(N-i), N-(i-1))} \hat{I}_{J(N-i), N-(i-1))}
\end{gathered}
$$

Here again, $V_{J(N-(i-1))}$ can be expressed as follows, and the voltage of the J-node, $I_{J(N-i),(N-(i-1)))}$ can be expressed again.

$$
\begin{gathered}
\hat{I}_{J(N-i),(N-(i-1))}=\left(\frac{\hat{S}_{J(N-(i-1))}}{\hat{V}_{J(N-(i-1))}}\right)^{*} \\
\hat{S}_{J(N-(i-1))}=\hat{S}_{J(N-(i-2))}+\left.Z_{J(N-(i-1), N-(i-2))}\left|\hat{I}_{J(N-(i-1), N-(i-2))^{2} \mid+S_{T(N-i)}}+Z_{T(N-i)}\right| I_{T(N-i)}\right|^{2} \mid \\
\hat{V}_{J(N-(i-1))}=\hat{V}_{J(N-(i-2))}+Z_{J(N-(i-1), N-(i-2))} \hat{I}_{J(N-(i-1), N-(i-2))}
\end{gathered}
$$

Here, if we re-express $S_{J(N-(i-2))}$ and $I_{J(N-(i-1), N-(i-2))}$

$$
\begin{gathered}
\hat{I}_{J(N-(i-1), N-(i-2))}=\left(\frac{\hat{S}_{J(N-(i-2))}}{\hat{V}_{J(N-(i-2))}}\right)^{*} \\
\hat{S}_{J(N-(i-2))}=S_{T(N-(i-2))}+Z_{T(N-(i-1)),(N-(i-2))}\left|I_{T(N-(i-1)),(N-(i-2))}\right|^{2}+S_{T(N-(i-1))} \\
+Z_{T(N-(i-1)}\left|I_{T(N-(i-1))}\right|^{2} \\
\hat{V}_{J(N-(i-2))}=V_{T(N-(i-2))}+Z_{T(N-(i-2))} I_{T(N-(i-2))}
\end{gathered}
$$

\section{References}

1. Rogers, K.M.; Klump, R.; Aquino-Lugo, H.K.A.A.; Overbye, T.J. An Authenticated Control Framework for Distributed Voltage Support on the Smart Grid. IEEE Trans. Smart Grid 2010, 1, 40-47. [CrossRef]

2. Turitsyn, K.; Šulc, P.; Backhaus, S.; Chertkov, M. Distributed control of reactive power flow in a radial distribution circuit with high photovoltaic penetration. In IEEE PES General Meeting, Minneapolis, MN, USA, 25-29 July 2010; IEEE: New York, NY, USA, 2010; pp. 1-6. Available online: http:/ / ieeexplore.iee.org/document/5589663/ (accessed on 10 July 2020).

3. Carvalho, P.M.S.; Correia, P.F.; Ferreira, L.A.F.M. Distributed Reactive Power Generation Control for Voltage Rise Mitigation in Distribution Networks. IEEE Trans. Power Syst. 2008, 23, 766-772. [CrossRef]

4. Masters, C.L. Voltage rise: The big issue when connecting embedded generation to long $11 \mathrm{kV}$ overhead lines. Power Eng. J. 2002, 16, 5-12. [CrossRef]

5. Keane, A.; Ochoa, L.F.; Vittal, E.; Dent, C.J.; Harrison, G.P. Enhanced Utilization of Voltage Control Resources with Distributed Generation. IEEE Trans. Power Syst. 2011, 26, 252-260. [CrossRef] 
6. Miyamoto, Y.; Hayashi, Y. valuating improved generation efficiency: One year using residential PV voltage control with a clustered residential grid-interconnected PV. In Proceedings of the 2012 3rd IEEE PES Innovative Smart Grid Technologies Europe, Berlin, Germany, 14-17 October 2012; pp. 1-8.

7. Hong, Y.; Luo, Y. optimal VAR Control Considering Wind Farms Using Probabilistic Load-Flow and Gray-Based Genetic Algorithms. IEEE Trans. Power Deliv. 2009, 24, 1441-1449. [CrossRef]

8. Villacci, D.; Bontempi, G.; Vaccaro, A. An adaptive local learning-based methodology for voltage regulation in distribution networks with dispersed generation. IEEE Trans. Power Syst. 2006, 21, 1131-1140. [CrossRef]

9. Hu, X.; Hu, H.; Verma, S.; Zhang, L.Z. Physics-Guided Deep Neural Networks for Power Flow Analysis. IEEE Trans. Power Syst. 2021, 36, 2082-2092. [CrossRef]

10. Yu, J.; Weng, Y.; Rajagopal, R. aToPa: A Data-Driven Parameter and Topology Joint Estimation Framework in Distribution Grids. IEEE Trans. Power Syst. 2002, 17, 1148-1153.

11. Guttromson, R.T. modeling distributed energy resource dynamics on the transmission system. IEEE Trans. Power Syst. 2018, 33, 4335-4347.

12. Nainar, K.; Iov, F. Smart Meter Measurement-Based State Estimation for Monitoring of Low-Voltage Distribution Grids. Energies 2020, 13, 5367. [CrossRef]

13. Manitsas, E.; Singh, R.; Pal, B.C.; Strbac, G. Distribution System State Estimation Using an Artificial Neural Network Approach for Pseudo Measurement Modeling. IEEE Trans. Power Syst. 2012, 27, 1888-1896. [CrossRef]

14. Aravindhababu, P.; Balamurugan, G. ANN based online voltage estimation. Appl. Soft Comput. 2012, 12, 313-319. [CrossRef]

15. Paucar, V.L.; Rider, M.J. Artificial neural networks for solving the power flow problem in electric power systems. Electr. Power Syst. Res. 2002, 62, 139-144. [CrossRef]

16. Serhat Berat, E.F.E.; Cebeci, M. Power flow analysis by Artificial Neural Network. Int. J. Energy Power Eng. 2013, 2, $204-208$.

17. Menke, J.-H.; Bornhorst, N.; Braun, M. Distribution system monitoring for smart power grids with distributed generation using artificial neural networks. Int. J. Electr. Power Energy Syst. 2019, 113, 472-480. [CrossRef]

18. Yang, Y.; Yang, Z.; Yu, J.; Zhang, B.; Zhang, Y.; Yu, H. Fast Calculation of Probabilistic Power Flow: A Model-Based Deep Learning Approach. IEEE Trans. Smart Grid 2020, 11, 2235-2244. [CrossRef]

19. Sekyung, H.; Kodaira, D.; Soohee, H.; Hasegawa, Y.; Aki, H. Automated impedance estimation method in low-voltage distribution network for coordinated voltage regulation. IEEE Trans. Smart Grid 2015, 7, 1012-1020. 